ARTICLE

DOI: $10.1038 / s 41467-017-00773-4$

\title{
Physical origins of current and temperature controlled negative differential resistances in $\mathrm{NbO}_{2}$
}

Suhas Kumar', Ziwen Wang ${ }^{2}$, Noraica Davila ${ }^{1}$, Niru Kumari ${ }^{1}$, Kate J. Norris ${ }^{1}$, Xiaopeng Huang ${ }^{1}$, John Paul Strachan ${ }^{1}$, David Vine ${ }^{3}$, A.L. David Kilcoyne (1) ${ }^{3}$, Yoshio Nishi ${ }^{2} \&$ R. Stanley Williams ${ }^{1}$

Negative differential resistance behavior in oxide memristors, especially those using $\mathrm{NbO}_{2}$, is gaining renewed interest because of its potential utility in neuromorphic computing. However, there has been a decade-long controversy over whether the negative differential resistance is caused by a relatively low-temperature non-linear transport mechanism or a high-temperature Mott transition. Resolving this issue will enable consistent and robust predictive modeling of this phenomenon for different applications. Here we examine $\mathrm{NbO}_{2}$ memristors that exhibit both a current-controlled and a temperature-controlled negative differential resistance. Through thermal and chemical spectromicroscopy and numerical simulations, we confirm that the former is caused by a $400 \mathrm{~K}$ non-linear-transport-driven instability and the latter is caused by the $1000 \mathrm{~K}$ Mott metal-insulator transition, for which the thermal conductance counter-intuitively decreases in the metallic state relative to the insulating state.

\footnotetext{
${ }^{1}$ Hewlett Packard Labs, 1501 Page Mill Road, Palo Alto, CA 94304, USA. ${ }^{2}$ Stanford University, 350 Serra Mall, Stanford, CA 94305, USA. ${ }^{3}$ Lawrence Berkeley National Laboratory, 1 Cyclotron Road, Berkeley, CA 94720, USA. Correspondence and requests for materials should be addressed to S.K. (email: Suhas.Kumar@hpe.com) or to R.S.W. (email: Stan.Williams@hpe.com)
} 
$\mathrm{N}$ egative differential resistance (NDR) in $\mathrm{NbO}_{2}$ is a manifestation of local activity that underlies threshold switching, generation of an action potential (in a neuristor), self-oscillations, and chaotic behavior, which are all being intensely researched for potential applications in neuromorphic or non-Boolean computing ${ }^{1-9}$. However, for several decades, there has been a controversy on whether the NDR is caused by a low-temperature (usually $<500 \mathrm{~K}$ ) non-linear-transport-driven thermal instability $4,10-12$ or a high-temperature Mott metal-insulator transition $(\mathrm{MIT})^{1,5,13-17}$. This issue is crucial for development of compact predictive models to study behaviors of larger computational systems constructed with NDR elements. Although accurate modeling of the low-current behavior consisting of the current-controlled NDR has been achieved recently $1,4,5,11,12$, a direct physicochemical measurement of the material temperature and localization effects during different NDR events have not been reported.

Here we examine nano and microscale $\mathrm{NbO}_{2}$ memristors that exhibit both a current-controlled and a less frequently observed temperature-controlled NDR. Through in operando thermoreflectance, in operando synchrotron X-ray transmission spectromicroscopy, transmission electron microscopy, and numerical simulations, we confirm that the current-controlled NDR is caused by a $\sim 400 \mathrm{~K}$ non-linear Poole-Frenkel-transportdriven instability and the temperature-controlled NDR is caused by the $\sim 1000 \mathrm{~K}$ Mott MIT. We highlight that temperature is the state variable for both types of NDRs, whereas they are driven by completely different physical mechanisms and occur at very different temperatures. We also show that the temperature and current density are spatially uniform over the memristor area through both NDRs when using a current source, whereas there is localized conduction channel/filament-formation when the memristor is biased in an unstable regime with a voltage source, recalling a long-standing and debated hypothesis ${ }^{18,19}$.

\section{Results}

Fabrication and transmission electron microscopy. We fabricated nanometer-scale $\mathrm{NbO}_{2}$ devices using a sub-100 nm diameter metallic TiN plug to contact a blanket thin film of amorphous $\mathrm{NbO}_{2}$ (Fig. la, b), the construction of which is described elsewhere ("Methods" section and Supplementary Figs. 2-4) ${ }^{11}$. The repeatable current-voltage curve (Fig. 1c) obtained by sweeping the applied current exhibits a region of current-controlled NDR ("NDR-1"), wherein the curve is single-valued for any value of current, and has been routinely observed in $\mathrm{NbO}_{2}$ before ${ }^{1,4}, 5,16$. However, at higher currents, this was followed by a rectangular hysteretic region consisting of a pair of sharp NDRs ("NDR-2"), which is neither current-controlled nor voltage-controlled. Current-voltage curves similar to the rectangular hysteresis have been observed in $\mathrm{VO}_{2}$ before and attributed to a Joule-heating-driven Mott MIT ${ }^{20}$. By studying the electron diffraction patterns of the initially amorphous active $\mathrm{NbO}_{2}$ layer across multiple devices, we observed irreversible crystallization only in devices that had been subject to current levels beyond those required to trigger NDR-2 (Fig. 1d). Since crystallization occurs at temperatures 21,22 in the range $800-1100 \mathrm{~K}$, this observation suggests that NDR-2 is related to the $\mathrm{MIT}^{23}, 24\left(T_{\mathrm{MIT}}\right.$ is in the range of $\left.1000-1100 \mathrm{~K}\right)$. Thus, NDR-1 was observed in both amorphous and crystalline $\mathrm{NbO}_{2}$, while NDR-2 was observed only in crystalline $\mathrm{NbO}_{2}$ (Supplementary Fig. 10).

Temperature mapping using thermoreflectance. In order to directly map the $\mathrm{NbO}_{2}$ temperature throughout the range of
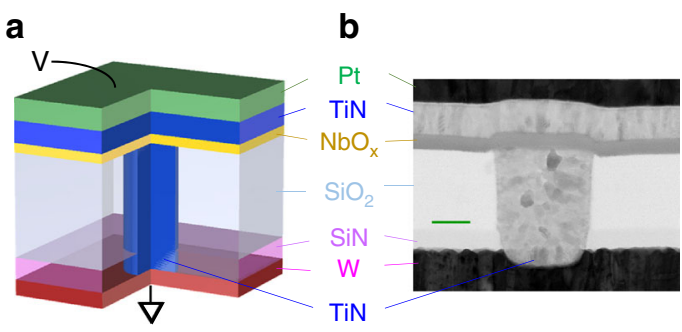

C

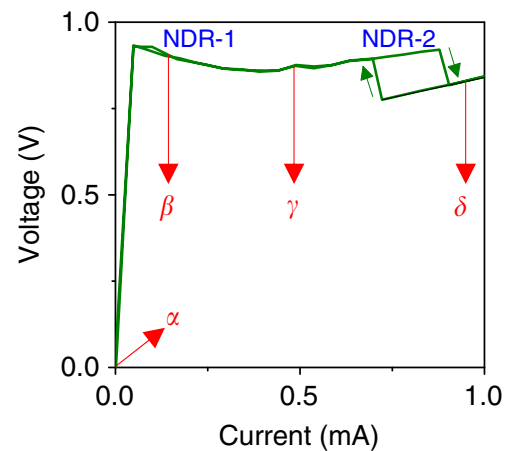

d
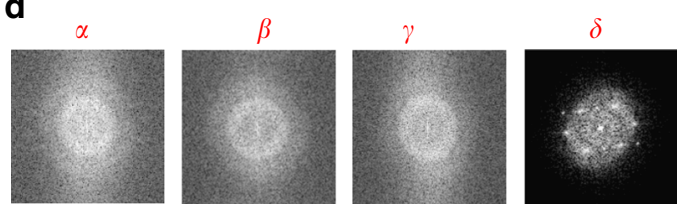

Fig. 1 Memristor structure, electrical behavior, and crystallization upon operation. a Sectional schematic of the device. b Cross-sectional transmission electron micrograph of a device identical to the one used here. Scale bar is $25 \mathrm{~nm}$. c A typical current-voltage curve obtained by sweeping current. Two regions containing negative differential resistance (NDR) behaviors, namely NDR-1 and NDR-2 are marked. $\alpha-\delta$ are current levels representative of: an unoperated device $(\alpha)$, onset of NDR-1 $(\beta)$, between NDR- 1 and NDR- $2(\gamma)$, and beyond NDR- $2(\delta)$. Electron diffraction patterns obtained using a transmission electron microscope within the active $\mathrm{NbO}_{2}$ layer on four different but nominally identical devices after operating them to different current levels (relative to NDR behaviors). Diffraction pattern at $\delta$ corresponds to a tetragonal [001] crystal projection, while the others were amorphous (as-grown)

current-voltage operation, we employed in operando synchronous time-multiplexed pump-probe thermoreflectance with a spatial resolution of $290 \mathrm{~nm}$ (using incident light of wavelength $530 \mathrm{~nm}$, Fig. $2 \mathrm{a})^{25}$. While the nanometer-scale devices described above were suitable for transmission electron microscopy measurements, we required different structures for the thermoreflectance and synchrotron X-ray measurements. For these, we fabricated $2.0 \times 2.5 \mu \mathrm{m}$ crosspoint devices on 150-nm-thick silicon nitride membranes suspended over holes etched into a silicon substrate, with a material stack consisting of $\mathrm{Pt}(15 \mathrm{~nm})$ (bottom electrode) $/ \mathrm{NbO}_{2}(15 \mathrm{~nm}) / \mathrm{TiN}(10 \mathrm{~nm})$ (top contact)/Pt $(15 \mathrm{~nm})$ (top electrode). These devices exhibited similar quasi-static electronic behavior to the nanometer-scale devices examined previously when driven with a current source ${ }^{26}$, but there was only a single pinched hysteresis loop that encompassed both NDR-1 and NDR-2 when the device was driven with a voltage source (Supplementary Fig. 1). The thermal isolation of the structure resulted in insignificant temperature gradients normal to the membrane/device surface during the Joule heating due to the electrical operation of the devices, thereby enabling the thermoreflectance measurements on the device stack (of thickness 
a

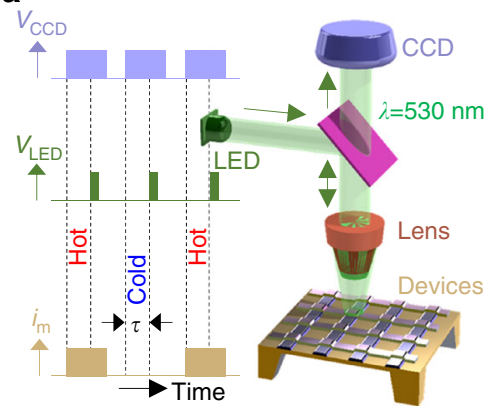

d

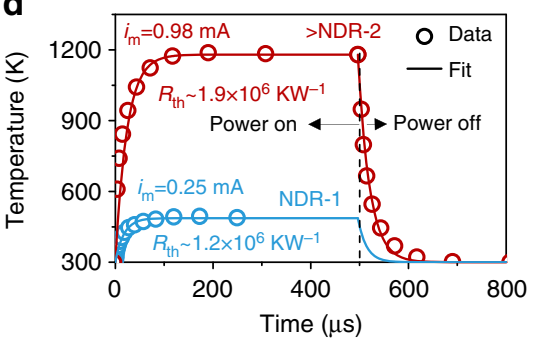

b

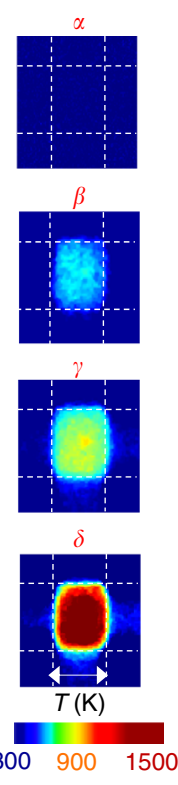

C

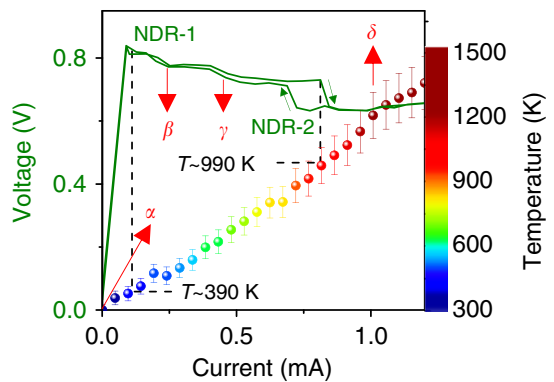

e

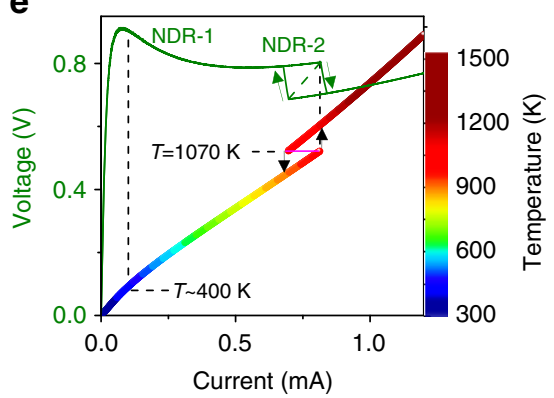

Fig. 2 In operando thermoreflectance. a Schematic of the experimental setup. Synchronous operational pulse signals to the charge coupled device (CCD) full-field camera $\left(V_{C C D}\right)$, light-emitting diode $(L E D)\left(V_{L E D}\right)$, and the crosspoint devices $\left(i_{m}\right)$ are shown. "Hot" and "cold" refer to durations in which there is a current applied and not applied to the device, respectively. $\tau$ is the delay-time between the pump (application of $\left.i_{\mathrm{m}}\right)$ and probe $\left(V_{\mathrm{LED}}\right.$ ). $\mathbf{b}$ Steady-state temperature maps of a single device at multiple constant current levels, with color-scale shown in c. Scale bar is $2 \mu \mathrm{m}$. c Experimental current-voltage curve of the device under measurement (using an increasing current sweep) and the corresponding average temperature within the crosspoint area at different current levels. Error bars represent the uncertainty in the measurement arising from calibration, noise, etc., (Supplementary Note 1). $\mathbf{d}$ Dynamic behavior of the average temperature within the crosspoint area of the same device upon application of a constant current (at time equal to 0 ) and upon withdrawal of the current (at time equal to $500 \mu \mathrm{s}$ ). e Simulated current-voltage curve and temperature of the device model (using increasing and decreasing current sweeps). Black arrows indicate hysteresis and temperature jumps predicted by the model. Pink solid line-segment indicates an abrupt jump in current that would occur upon a parametric temperature sweep. Green dashed-line segment is an abrupt jump in the current-voltage curve upon a parametric temperature sweep. Black dashed lines in $\mathbf{c}$, e are used to indicate the temperature at the onset of the two regions of negative differential resistances (NDRs), namely, NDR-1 and NDR-2

$<50 \mathrm{~nm}$ ) to accurately represent the temperature of the active $\mathrm{NbO}_{2}$ layer ${ }^{27}$ (Supplementary Notes 1 and 2, Supplementary Figs. 6 and 7). The temperature maps (Fig. 2b) and the corresponding average temperatures within the crosspoint area (Fig. 2c) at different current levels (obtained using a current source) reveal that the temperature at the onset of NDR-1 is $\sim 400 \mathrm{~K}$, and that around NDR-2 is $\sim 1000 \mathrm{~K}$, which coincides with the $\mathrm{MIT}^{1,5}, 13-17$. The temperature distribution within most of the crosspoint area (Fig. 2b) was spatially uniform across the entire current range, which indicates that the current density is also uniform. To estimate the thermal resistance of the device structure, we measured the temporal temperature evolution (rise and decay) within the crosspoint after application and withdrawal of electrical power (Fig. 2d). We then fitted the data to the dynamical equation for the state variable, which is the temperature (T), i.e., Newton's law of cooling (Eq. 1) expressed in terms of the memristor current $\left(i_{\mathrm{m}}\right)$, voltage $\left(v_{\mathrm{m}}\right)$, and lumped thermal properties ${ }^{11}$,

$$
\frac{\mathrm{d} T}{\mathrm{~d} t}=\frac{i_{\mathrm{m}} v_{\mathrm{m}}}{C_{\mathrm{th}}}-\frac{T-T_{\mathrm{amb}}}{C_{\mathrm{th}} R_{\mathrm{th}}(T)},
$$

where $T_{\mathrm{amb}}$ is the ambient temperature of $300 \mathrm{~K}$; $C_{\mathrm{th}}$ is the thermal capacitance; and $R_{\text {th }}$ is the effective thermal resistance. We determined that $R_{\mathrm{th}}$ for a steady-state temperature of $\sim 490 \mathrm{~K}$ was $1.4 \times 10^{6} \pm 0.2 \mathrm{KW}^{-1} \quad\left(T<T_{\mathrm{MIT}}\right.$, current levels within NDR-1) and that for a steady-state temperature of $>1100 \mathrm{~K}(T>$ $T_{\text {MIT, }}$ current levels above NDR-2) was $1.9 \times 10^{6} \pm 0.3 \mathrm{KW}^{-1}$ (while we used the same $C_{\mathrm{th}}$ of $1.25 \times 10^{-11} \mathrm{WsK}^{-1}$ in both cases).
While these estimates are obtained by crude simplifications of the values and functional forms of $R_{\mathrm{th}}$ and $C_{\mathrm{th}}$, they nonetheless agreed well with experimental data and provide a good starting point for further detailed modeling.

Numerical modeling. To model the experimentally observed quasi-static current-voltage behavior, we adapt and extend a recently proposed modified three-dimensional Poole-Frenkel conduction mechanism with temperature as the state variable, represented by Eq. $2^{4,11}$.

$$
i_{\mathrm{m}}=\left[\sigma_{0} e^{-\frac{E_{\mathrm{a}}}{k_{\mathrm{B}} T}} A\left\{\left(\frac{k_{\mathrm{B}} T}{\omega}\right)^{2}\left(1+\left(\frac{\omega \sqrt{v_{\mathrm{m}} / d}}{k_{\mathrm{B}} T}-1\right) \mathrm{e}^{\frac{\omega \sqrt{v_{\mathrm{m}} / d}}{k_{\mathrm{B}} T}}\right)+\frac{1}{2 d}\right\}\right] v_{\mathrm{m}},
$$

where $A$ is the lateral device area; $k_{\mathrm{B}}$ is the Boltzmann constant (in $\mathrm{eV}$ ), $d$ is the thickness of $\mathrm{NbO}_{2}$, and $\sigma_{0}, E_{\mathrm{a}}$, and $\omega$ are material property constants described elsewhere ${ }^{11}$. To account for the MIT, following the results from the dynamic temperature measurements, we introduced an abrupt change in $R_{\mathrm{th}}: 1.4 \times 10^{6}$ (for $T<T_{\mathrm{MIT}}$ ) and $1.9 \times 10^{6}$ (for $T \geq T_{\mathrm{MIT}}$ ), where $T_{\mathrm{MIT}}=1070 \mathrm{~K}$. The quasi-static behavior described by Eqs. 1 and 2 (with $\frac{\mathrm{d} T}{\mathrm{~d} t}=0$ ) is in good agreement with experimentally measured data, especially in the reproduction of NDR-1 and NDR-2 (Fig. 2e). Significant increases in the complexity of the model are required to yield only moderate improvements in agreement with the experimental data, so we opt for simplicity here. The simulated temperatures of $\mathrm{NbO}_{2}$ across the applied current range are also in good agreement with the experimental measurements (Fig. 2c, e). 

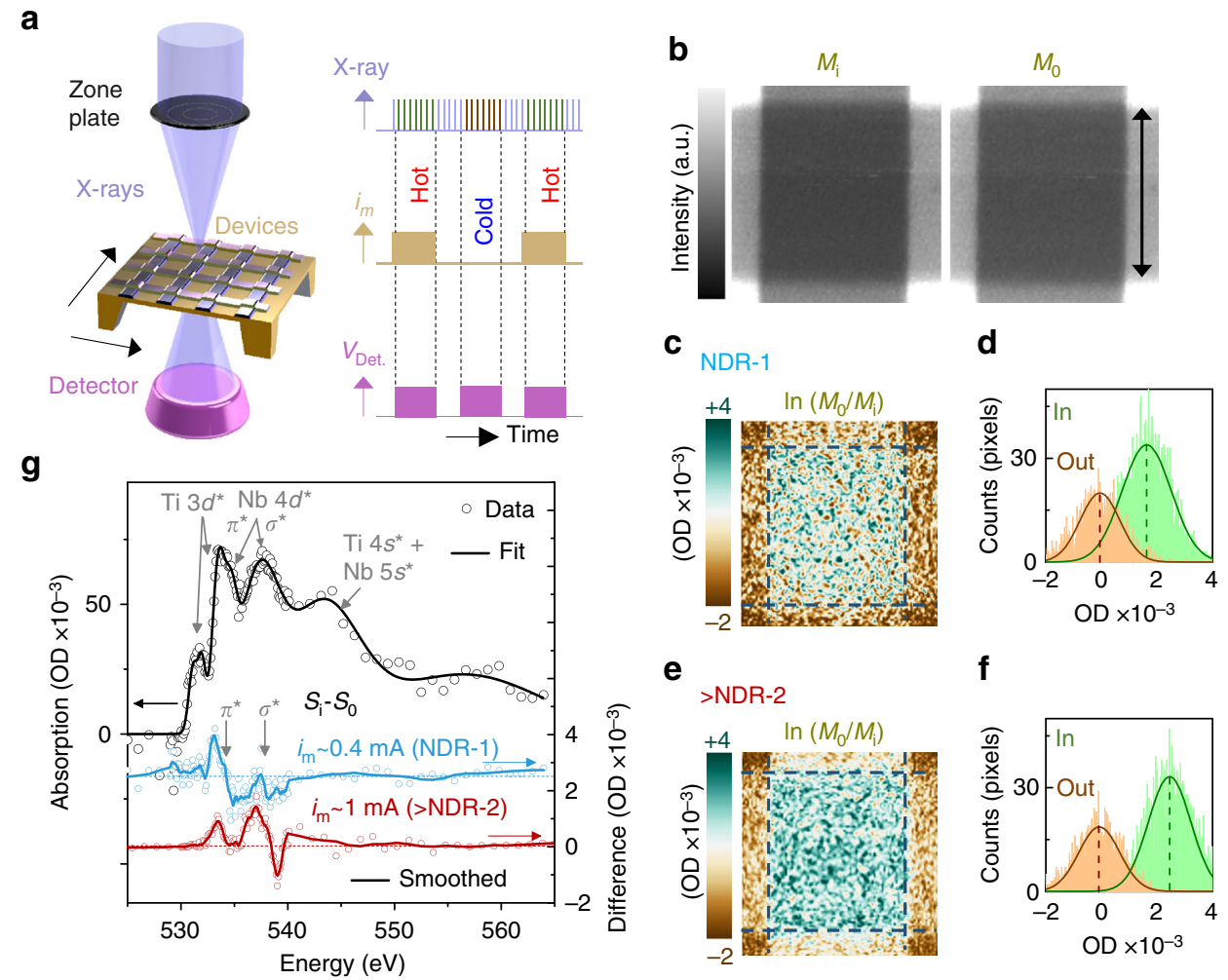

Fig. 3 In operando synchrotron X-ray spectromicroscopy. a Schematic of the experimental setup. Synchronous operational pulse signals to the detector and the devices are shown, along with the incoming asynchronous $\mathbf{X}$-ray pulses. $\mathbf{b} \mathbf{X}$-ray transmission maps of the crosspoint area of a fresh device identical to the one used in Fig. 2, with current ( 0.4 mA, sufficient to cause NDR-1) $\left(M_{\mathrm{i}}\right)$ and with no current $\left(M_{0}\right)$ obtained using $533.2 \mathrm{eV} X$-rays. Scale bar is $2 \mu \mathrm{m}$. c Logarithmic ratio of the maps in $\mathbf{b}$. Intensity in optical density, OD. $\mathbf{d}$ Histograms of the data inside and outside the crosspoint area in c. e Logarithmic ratio of maps similar to those in (b) obtained with a current sufficient to exceed NDR-2 $(\sim 1 \mathrm{~mA})$ and, (f) its corresponding histograms, similar to those in (d). $\mathbf{g}$ X-ray absorption spectra of the material within the crosspoint, along with the component bands marked. The spectral differences between the material with current and that with no current are also shown for two different currents, as marked (arbitrarily offset, horizontal dashed lines indicate zero difference for respective colors)

The predicted hysteretic jumps during current-source operation across NDR-2 (black arrows) are too small to be observed experimentally, given the measurement uncertainty. They would change to a single-valued function of temperature (pink solid line) upon a parametric $T$ sweep as displayed in Fig. 2e. Thus, NDR-2 is a temperature-controlled instability that manifests as a rectangular hysteresis during current-source operation. This behavior is similar to the Chua Corsage current-voltage characteristic $^{28}$. Further, the counter-intuitive increase in $R_{\mathrm{th}}$ in the metallic state $\left(T>T_{\mathrm{MIT}}\right)$ relative to the insulating/semiconducting state $\left(T<T_{\mathrm{MIT}}\right)$, which is consistent for both temperature and electrical measurements (Supplementary Note 3 , Supplementary Figs. 8 and 11), is opposite to that expected from the Wiedemann-Franz law. A recent report also described such anomalous behavior of $R_{\mathrm{th}}$ across the Mott transition in $\mathrm{VO}_{2}$ and provided plausible explanations that may cover the behavior observed here as well, although a comprehensive theory remains unexplored $^{29}$.

Synchrotron X-ray spectromicroscopy. To further analyze the uniformity and the chemical nature of the $\mathrm{NbO}_{2}$ material changes in operando, we employed synchronous time-multiplexed scanning transmission X-ray microscopy (STXM) (Fig. 3a, Supplementary Note 4, Supplementary Fig. 12) using synchrotron radiation, which has been described elsewhere ${ }^{30,} 31$. This technique provided a spatial resolution of $<30 \mathrm{~nm}$ and spectral resolution of $70 \mathrm{meV}$ using X-ray energies tuned to the $\mathrm{O}$ K-edge, and the synchronous measurements enabled the detection of very low signal differences ${ }^{26,30}$. For these experiments, we were able to use devices that were identical to the ones used for thermoreflectance, wherein the suspended thin silicon nitride membrane enabled transmitted X-ray detection. The X-ray maps of the crosspoint area (Fig. 3b) without a current $\left(M_{0}\right)$ and that with a current sufficient to cause NDR-1 $\left(M_{\mathrm{i}}\right)$ do not show any noticeable differences between each other by eye. However, the logarithmic ratio of the maps (representing the optical density, OD) displays a small but detectable signal that appears to be uniform over the crosspoint area (Fig. 3c) and upshifted to higher values with respect to the material outside, revealing a chemical or electronic response to the flow of current near NDR-1. Both OD distributions were essentially Gaussian with means shifted by slightly $<2 \times 10^{-3}$ and essentially identical standard deviations $\left(S \approx 0.73 \times 10^{-3}\right)$, confirming that the changes were uniform. The reported data were averaged over several thousand measurement cycles, so the shift in the means is statistically significant ${ }^{30}$. A current sufficient to exceed NDR-2 caused a larger uniform shift of the $\mathrm{OD}$ to higher values (Fig. 3e, f, Supplementary Fig. 5, Supplementary Note 6). The O K-edge spectral difference between the material within the crosspoint with no current and that with a current sufficient to cause NDR-1 (Fig. 3g) displays a prominent feature at the rising edge of the $\pi^{*}$ band of $\mathrm{NbO}_{2}{ }^{32,33}$, consistent with a downshifting of the lowest conduction band ("Methods" section). While the physical origin of this feature is uncertain, it could be caused by a lattice expansion of the $\mathrm{NbO}_{2}$ due to Joule heating at NDR-1 4,11 . Also, the less pronounced signal in the difference spectrum 

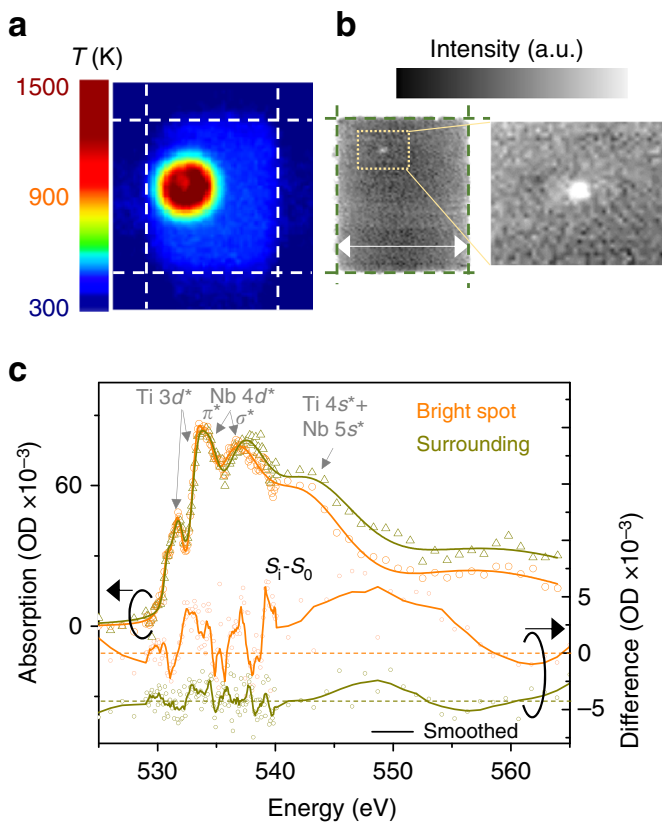

Fig. 4 Operation with a voltage source. a Temperature map of a fresh crosspoint device identical to the ones explained previously held at an applied voltage of $1 \mathrm{~V}$ with $R_{\mathrm{S}}<R_{\mathrm{NDR}}$ ). $\mathbf{b} \mathrm{X}$-ray transmission maps of a fresh crosspoint device identical to the one used in (a) that was previously subject to an applied voltage of $1 \mathrm{~V}$ (no bias was applied during acquisition of this data). Scale bar is $2 \mu \mathrm{m}$. A localized bright spot is shown in a magnified map. c X-ray absorption spectra of the "bright spot" and its "surrounding" region, color-coded to the legend. The spectral differences between the material with current and that with no current $\left(S_{i}-S_{0}\right)$ are also shown for the two different regions (arbitrarily offset, horizontal dashed lines indicate zero difference for respective colors)

corresponding to the Ti-O bond energies likely arise from a non-linear conduction mechanism in the $\mathrm{TiO}$ formed at the interface of TiN and $\mathrm{NbO}_{2}$ (Supplementary Figs. 2 and 3 ) $^{34}$. Beyond NDR-2, a similarly obtained spectral difference displays prominent features consistent with downshifting of both the $\pi^{\star}$ and $\sigma^{\star}$ bands, in agreement with theoretical predictions of an increased conductivity at the MIT and an accompanying Peierls distortion in the crystal structure ${ }^{24}$. The Mott + Peierls transitions in $\mathrm{NbO}_{2}$ responsible for NDR-2 are similar to those in $\mathrm{VO}_{2}$ causing a behaviorally similar $\mathrm{NDR}^{29,}, 35$ (discussed further in Supplementary Note 5 and Supplementary Fig. 13).

Filament formation using a voltage source. Following the observations of spatially uniform temperature and chemical changes during current-source measurements, we studied such responses when the devices were biased directly by a voltage source (with no external series resistance to limit the current; there was a series electrode resistance of about $300 \Omega$ ). Upon applying $1 \mathrm{~V}$ pulses (estimated current higher than $1.5 \mathrm{~mA}$ from the current-voltage sweeps shown in Supplementary Fig. 1, and thereby having accessed both the NDRs) to a crosspoint device using the thermoreflectance apparatus, we observed a localized hot-spot in the temperature map (Fig. 4a) that exhibited a temperature of more than $1200 \mathrm{~K}$, while the rest of the crosspoint area had a relatively uniform temperature of $\sim 400 \mathrm{~K}$ (corresponding to the onset of NDR-1). In a similar voltagecontrolled experiment on a fresh but identical device probed using the time-multiplexed STXM technique, we observed the formation of a localized conduction channel, roughly $100 \mathrm{~nm}$ across, which persisted even after the voltage was removed, likely due to irreversible stoichiometry changes caused by the high temperatures (Fig. 4b, Supplementary Fig. 9). The post-O K-edgeabsorption intensity within the channel was lower relative to its surroundings (Fig. 4c), revealing a lower $\mathrm{O}$ concentration. At the $\mathrm{O}$ K-edge, significant downshifting in energy of the component bands of $\mathrm{NbO}_{2}$ within the channel also indicated chemical reduction of the oxide ${ }^{32,33}$. The spectral differences between the material with a $1 \mathrm{~V}$ and with zero bias also revealed a higher current density inside the channel ${ }^{26}$. The formation of a high current density "filament" or channel in a background of lower current density resulting from a symmetry-breaking instability in the NDR region was first proposed by Ridley based on entropy-production minimization arguments ${ }^{18}$, which were later questioned by Landauer ${ }^{19}$. Numerical simulations of NDR by Funck et al. ${ }^{4}$ also showed a high-current density channel within a lower-current density region, but this was enforced by the cylindrical symmetry of the model and the radial boundary conditions for heat flow. In our voltage source experiments, the $\mathrm{NbO}_{2}$ experienced irreversible material changes due to the high temperatures that were reached, so an unambiguous determination of the mechanism responsible for the channel formation is not possible from our data. Resolution of this interesting and important issue that is critical for robust modeling of NDR and other electrical instabilities will require further research.

\section{Discussion}

Using spectromicroscopic characterization techniques to measure temperature and chemical spatial distributions, along with numerical simulations, we confirmed that the current-controlled NDR-1 in $\mathrm{NbO}_{2}$ is caused by a Joule-heating-driven thermal runaway in strongly nonlinear conduction at relatively low temperatures $(\sim 400 \mathrm{~K})$ and further demonstrated that NDR-2 is a temperature-controlled MIT-driven hysteresis at high temperature $(\sim 1000 \mathrm{~K})$. Both types of NDR within the same material are Joule-heating driven, and thus have $T$ as a state variable, but they are caused by distinctly different physical mechanisms at very different temperatures. In addition, we showed that using a current source yields, a spatially uniform current density over the device area, while operating the devices with a voltage source causes the formation of a localized high current density channel, highlighting an outstanding problem on the subject of filament formation during electrical instabilities. These results definitively resolve the question of whether the NDR of $\mathrm{NbO}_{2}$ is caused by nonlinear temperature-dependent conduction or by a MIT-the answer is both mechanisms are distinct and present, which provides a rich set of nonlinear behaviors for potential exploitation.

\section{Methods}

Film growth. $\mathrm{Si}_{3} \mathrm{~N}_{4}$ of $150 \mathrm{~nm}$ was grown using low-pressure chemical vapor deposition on double side-polished $\mathrm{Si}$ wafer with low $\mathrm{p}$-doping. Holes were etched into the $\mathrm{Si}$ wafer to allow free suspension of $\mathrm{Si}_{3} \mathrm{~N}_{4}$ membranes. Crosspoint cells were lithographically patterned onto these membranes. The Pt electrodes were evaporated from a Pt target. The layers of $\mathrm{NbO}_{2}$ and TiN were sputter-deposited.

Spectral processing. Spectra were first normalized to background absorptions in the vacuum chamber, measured through a blank $\mathrm{Si}_{3} \mathrm{~N}_{4}$ membrane (with no other material on it). The resulting spectra were corrected for a linear background in the pre-absorption-edge region. Peak fitting was done using the software Sigmaplot PeakFit. The pre-edge of the oxygen K-edge was aligned to zero, a linear background in the pre-edge region was subtracted, and the data was smoothed by $1 \%$ using Savitzky-Golay smoothing. The peaks were composed of a convolution of a Lorentzian component (to account for the broadening of spectral lines due to excited electron lifetime) and a Gaussian component (to account for the resolution of the spectrometer, mostly due to the beamline monochromator). For each spectrum, the component bands were allowed to vary in width, amplitude, and 
position to obtain the best fit. Smoothing of data in Figs. 3 and 4 was performed using Savitzky-Golay smoothing over 15 adjacent points.

Data availability. The data that support the findings of this study are available from the corresponding author upon reasonable request.

Received: 6 April 2017 Accepted: 26 July 2017

Published online: 22 September 2017

\section{References}

1. Pickett, M. D., Medeiros-Ribeiro, G. \& Williams, R. S. A scalable neuristor built with Mott memristors. Nat. Mater. 12, 114-117 (2012).

2. Chang, T., Yang, Y. \& Lu, W. Building neuromorphic circuits with memristive devices. IEEE Circuits Syst. Mag. 13, 56-73 (2013).

3. Moon, K. et al. in 2016 International Symposium on VLSI Technology, Systems and Application (VLSI-TSA) 1-2 (IEEE, 2016).

4. Funck, C. et al. Multidimensional simulation of threshold switching in $\mathrm{NbO}_{2}$ based on an electric field triggered thermal runaway model. Adv. Electron. Mater. 2, 1600169 (2016).

5. Liu, X., Li, S., Nandi, S. K., Venkatachalam, D. K. \& Elliman, R. G. Threshold switching and electrical self-oscillation in niobium oxide films. J. Appl. Phys. 120, 124102 (2016).

6. Pickett, M. D. \& Williams, R. S. Sub-100 fJ and sub-nanosecond thermally driven threshold switching in niobium oxide crosspoint nanodevices. Nanotechnology. 23, 215202 (2012).

7. Mainzer, K. \& Chua, L. Local Activity Principle (Imperial College Press, 2013).

8. Chua, L. Memristor, Hodgkin-Huxley, and edge of chaos. Nanotechnology 24, 383001 (2013).

9. Kumar, S., Strachan, J. P. \& Williams, R. S. Chaotic dynamics in nanoscale $\mathrm{NbO}_{2}$ Mott memristors for analogue Computing. Nature 548, 318-321 (2017).

10. Shin, S. H., Halpern, T. \& Raccah, P. M. High-speed high-current field switching of $\mathrm{NbO}_{2}$. J. Appl. Phys. 48, 3150-3153 (1977).

11. Gibson, G. A. et al. An accurate locally active memristor model for S-type negative differential resistance in $\mathrm{NbO}_{\mathrm{x}}$. Appl. Phys. Lett. 108, 023505 (2016).

12. Slesazeck, S. et al. Physical model of threshold switching in $\mathrm{NbO}_{2}$ based memristors. RSC Adv. 5, 102318-102322 (2015).

13. Vezzoli, G. C. Recovery curve for threshold-switching $\mathrm{NbO}_{2}$. J. Appl. Phys. 50, 6390-6395 (1979)

14. Zhou, Y. \& Ramanathan, S. Mott memory and neuromorphic devices. Proc. IEEE 103, 1289-1310 (2015).

15. Murdoch, B. J. et al. Memristor and selector devices fabricated from $\mathrm{HfO}_{2}-\mathrm{xNx}$. Appl. Phys. Lett. 108, 143504 (2016).

16. Cha, E. et al. Comprehensive scaling study of $\mathrm{NbO}_{2}$ insulator-metal-transition selector for cross point array application. Appl. Phys. Lett. 108, 153502 (2016).

17. Kim, K. M. et al. Low power, self-rectifying, and forming-free memristor with an asymmetric programing voltage for a high density crossbar application. Nano. Lett. 11, 6724-6732 (2016)

18. Ridley, B. K. Specific negative resistance in solids. Proc. Phys. Soc. Lond. 82, 954-966 (1963).

19. Landauer, R. Stability and entropy production in electrical circuits. J. Stat. Phys. 13, 1-16 (1975)

20. Kumar, S. et al. Local temperature redistribution and structural transition during Joule-heating-driven conductance switching in $\mathrm{VO}_{2}$. Adv. Mater. 25, 6128-6132 (2013)

21. Zhang, J. et al. Thermally induced crystallization in $\mathrm{NbO}_{2}$ thin films. Sci. Rep. 6, 34294 (2016).

22. Snook, M. W. G., Thomas, C. B. \& Reehal, H. S. Preparation of polycrystalline films of $\mathrm{NbO}_{2}$ by rf sputtering. Mater. Lett. 3, 462-466 (1985).

23. Chudnovskii, F. A., Odynets, L. L., Pergament, A. L. \& Stefanovich, G. B. Electroforming and switching in oxides of transition metals: the role of metal-insulator transition in the switching mechanism. J. Solid State Chem. 122, 95-99 (1996).

24. Eyert, V. The metal-insulator transition of $\mathrm{NbO}_{2}$ : an embedded Peierls instability. Europhys. Lett. 58, 851 (2002).

25. Farzaneh, M. et al. CCD-based thermoreflectance microscopy: principles and applications. J. Phys. D Appl. Phys. 42, 143001 (2009).

26. Kumar, S. et al. Direct observation of localized radial oxygen migration in functioning tantalum oxide memristors. Adv. Mater. 28, 2772-2776 (2016).
27. Cai, W. et al. Thermal transport in suspended and supported monolayer graphene grown by chemical vapor deposition. Nano. Lett. 10, 1645-1651 (2010).

28. Mannan, Z. I., Choi, H. \& Kim, H. Chua corsage memristor oscillator via Hopf bifurcation. Int. J. Bifurc. Chaos 26, 1630009 (2016).

29. Lee, S. et al. Anomalously low electronic thermal conductivity in metallic vanadium dioxide. Science 355, 371-374 (2017).

30. Kumar, S. et al. In-operando synchronous time-multiplexed O K-edge X-ray absorption spectromicroscopy of functioning tantalum oxide memristors. J. Appl. Phys. 118, 034502 (2015).

31. Kilcoyne, A. L. et al. Interferometer-controlled scanning transmission X-ray microscopes at the advanced light source. J. Synchrotron Radiat. 10, 125-136 (2003).

32. Soriano, L. et al. The $\mathrm{O} 1 \mathrm{~s} \mathrm{X}$-ray absorption spectra of transition-metal oxides: the $\mathrm{TiO}_{2}-\mathrm{ZrO}_{2}-\mathrm{HfO}_{2}$ and $\mathrm{V}_{2} \mathrm{O}_{5}-\mathrm{Nb}_{2} \mathrm{O}_{5}-\mathrm{Ta}_{2} \mathrm{O}_{5}$ series. Solid State Commun. 87, 699-703 (1993)

33. Tao, R. et al. Electron energy-loss spectroscopy study of metallic $\mathrm{Nb}$ and $\mathrm{Nb}$ oxides. J. Appl. Phys. 110, 124313 (2011).

34. Alexandrov, A. S. et al. Current-controlled negative differential resistance due to Joule heating in $\mathrm{TiO}_{2}$. Appl. Phys. Lett. 99, 202104 (2011).

35. Kumar, S. et al. Sequential electronic and structural transitions in $\mathrm{VO}_{2}$ observed using X-ray absorption spectromicroscopy. Adv. Mater. 26, 7505-7509 (2014).

\section{Acknowledgements}

The research is in part based upon work supported by the Office of the Director of National Intelligence (ODNI), Intelligence Advanced Research Projects Activity (IARPA), via contract number 2017-17013000002. X-ray experiments were performed at the Advanced Light Source in Lawrence Berkeley National Laboratory, a US DOE Office of Science User Facility under contract no. DE-AC02-05CH11231. Work was performed in part at the Stanford Nanofabrication Facility that is supported by National Science Foundation through the NNIN under Grant ECS-9731293.

\section{Author contributions}

S.K., Z.W., J.P.S. and R.S.W. defined the problem and conceptualized the experimental approaches to possible solutions. Z.W and N.D. fabricated the devices with assistance from S.K. S.K. and N.K. performed the thermoreflectance measurements. S.K., Z.W., N.K. and X.H. performed the synchrotron X-ray studies with assistance from N.D., D.V. and A.L.D.K. K.J.N. performed the electron microscopy measurements. S.K. and R.S.W. performed the numerical simulations. Y.N. supervised the work done by Z.W. All authors provided comments on the data and their interpretations. S.K., Z.W. and R.S.W. wrote the manuscript.

\section{Additional information}

Supplementary Information accompanies this paper at doi:10.1038/s41467-017-00773-4

Competing interests: The authors declare no competing financial interests.

Reprints and permission information is available online at http://npg.nature.com/ reprintsandpermissions/

Publisher's note: Springer Nature remains neutral with regard to jurisdictional claims in published maps and institutional affiliations.

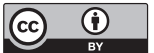

Open Access This article is licensed under a Creative Commons Attribution 4.0 International License, which permits use, sharing, adaptation, distribution and reproduction in any medium or format, as long as you give appropriate credit to the original author(s) and the source, provide a link to the Creative Commons license, and indicate if changes were made. The images or other third party material in this article are included in the article's Creative Commons license, unles indicated otherwise in a credit line to the material. If material is not included in the article's Creative Commons license and your intended use is not permitted by statutory regulation or exceeds the permitted use, you will need to obtain permission directly from the copyright holder. To view a copy of this license, visit http://creativecommons.org/ licenses/by/4.0/

(C) The Author(s) 2017 